\title{
CHARACTERIZATIONS OF SUPPORTS OF BALAYAGED MEASURES
}

\author{
MASAYUKI ITÔ
}

\section{Introduction}

Beurling and Deny [1], [2] introduced Dirichlet spaces by generalizing the notion of "energy" in potential theory. They showed the existences of balayaged measures and condensor measures in the theory of Dirichlet spaces. The purpose of this paper is to characterize the supports of those measures. First we obtain the following result.

Let $D$ be a Dirichlet space on a locally compact Hausdorff space $X$, and let $F$ be a regular set in $X \times X$ containing the diagonal set of $X \times X$. Then the following two conditions are equivalent.

(1) For any pure potential $u_{i}$ in $D$ and any closed neighborhood $\omega$ of the support of $\mu$, the support of the balayaged measure of $\mu$ to $\mathscr{C} \omega$ is contained in $F_{w} \cap \overline{\mathscr{C} \omega}$.

(2) The support of the singular measure of $D$ is contained in $F$.

In the above statement, $F_{\omega}$ means the projection of $(\omega \times X) \cap F$ to $X$.

Furthermore we shall examine a relation between the supports of condensor measures and the support of the singular measure of $D$.

Next we shall consider special Dirichlet spaces on the $n$-dimensional Euclidean space $R^{n}(n \geq 1)$. With a special Dirichlet space $D$ associates a real valued negative definite function $\lambda$ such that $\lambda^{-1}$ is locally summable, and the norm in $D$ is explicitely represented. By Levy-Khintchine's theorem, $\lambda(x)$ is as follows :

$$
\lambda(x)=C+Q(x)+\int_{|y|>0}\left(1-e^{2 \pi i x \cdot y}\right) d \sigma_{1}(y),
$$

where $C$ is a non-negative constant, $Q(x)$ is a positive quardratic form and $\sigma_{1}$ is a positive symmetric measure in $R^{n}-\{0\}$. Then we shall obtain a more

Received April 18, 1966. 
precise result about the relation among the supports of balayaged measures, of condensor measures and of the above-mentioned $\sigma_{1}$.

\section{Preliminaries on Dirichlet spaces}

To begin with, we shall give the definition of Dirichlet spaces according to Beurling and Deny [2].

Definition 1.1) A transformation $T$ on the complex plane $(5$ into itself is called a normal contraction if the following conditions are satisfied:

$$
T(0)=0 \text { and }\left|T z_{1}-T z_{2}\right| \leq\left|z_{1}-z_{2}\right|
$$

for each couple $z_{1}, z_{2}$ in 5 .

Let $X$ be a locally compact Hausdorff space. Let $C_{K}$ be the space of complexvalued continuous functions with compact support provided with the topology of uniform convergence. We denote by $C_{K}^{+}$the totality of positive functions in $C_{K}$.

Definition 2." Let $\xi$ be a positive Radon measure in $X$ which is everywhere dense (i.e., $\xi(\omega)>0$ for each non-empty open set $\omega$ in $X$ ). A Hilbert space $D=D(X, \xi)$ is called a $\xi$-Dirichlet space (simply, a Dirichlet space) if each element in $D$ is a complex-valued function $u(x)^{3)}$ which is locally summable for $\xi$ and the following three conditions are satisfied:

a) For each compact subset $K$ in $X$, there exists a positive number $A(K)$ such that

$$
\int_{\mathbf{K}} u(x) \mid d \xi(x) \leq A(K)\|u\|
$$

for any $u$ in $D$.

b) $C_{K} \cap D$ is dense both in $C_{K}$ and $D$.

c) For any normal contraction $T$ and any $u$ in $D$,

$$
T u \in D \text { and }\|T u\| \leq\|u\| \text {. }
$$

Now we shall define potentials in the Dirichlet space $D$.

1) Cf. [2], p. 209.

2) Cf. [2], p. 209.

3) We take the usual liberty of speaking of functions when actually equivalence classes are meant. 
Definition 3.4) An element $u$ in $D$ is called a potential if there exists a Radon measure $\mu_{\text {. in } X \text { satisfying }}$

$$
(u, v)=\int v(x) d \mu(x)
$$

for any $v$ in $C_{K} \cap D$. We denote it by $u_{\mu}$. Especially if $\mu$ is positive, $u_{\mu}$ is called a pure potential.

The space of linear combinations of pure potentials is dense in $D$. This definition of potentials leads to a kernel-free potential theory. Next we shall give the definition of the spectrum of an element in $D$.

Given an element $u$ in $D$, there exists the greatest open set $\omega$ having the following property :

$$
(u, v)=0
$$

for any $v$ in $C_{K} \cap D$ with support in $\omega$.

Definition 4.5) The complementary set of such an open set is called the spectrum of $u$, denoted by $\subseteq(u)$.

Obviously $\mathcal{S}\left(\mathfrak{u}_{\mu}\right)=S_{u}{ }^{6}$.

The next four theorems obtained by Beurling and Deny [2] are essential.

Beurling and Deny's Condensor Theorem ${ }^{7}$. Let $\omega_{1}$ and $\omega_{0}$ be two open sets in $X$ with disjoint closures, $\omega_{1}$ being relatively compact. Then there exists a potential $u_{\mu}$ generated by a real measure $\mu$ such that

$$
\begin{array}{ll}
\text { (C. 1) } & 0 \leq u_{\mu}(x) \leq 1 \text { p.p. in } X^{s)}, \\
\text { (C. 2) } & u_{\mu}(x)=1 \text { p.p. in } \omega_{1} \text { and } u_{\mu}(x)=0 \text { p.p. in } \omega_{0}, \\
\text { (C. 3) } & \mu^{+} \text {is supported by } \overline{\omega_{1}} \text { and } \mu^{-} \text {is supported by } \bar{\omega}_{0} .
\end{array}
$$

We shall say that the measure $\mu$ is a condensor measure associated with $\omega_{1}$ and $\omega_{0}$. Let $\omega_{0}$ be the empty set. Then we get the equilibrium theorem.

Beurling and Deny's Balayage Theorem". For a pure potential $u_{\mu}$ and an

4) Cf. [2], p. 209 .

5) Cf. [2], p. 214 .

6) $S_{\mu}$ is the support of $\mu$.

7) Cf. [2], p. 210 and [4], p. 4.

8) A property is said to hold p.p. in a subset $E$ in $X$ if the property holds in $E$ except a set which is locally of $\xi$-measure 0 .

9) Cf. [2], p. 210 and [4], p. 6. 
open set $\omega$, there exists a pure potential $u_{\mu^{\prime}}$ satisfying the following conditions:

(B. 1) The measure $\mu^{\prime}$ is supported by $\bar{\omega}$ and $\int d \mu^{\prime} \leq \int d \mu$.

(B. 2) $\quad u_{\mu^{\prime}}(x)=u_{\mu}(x)$ p.p. in $\omega$.

(B. 3) $\quad u_{\mu}(x) \leq u_{\mu}(x)$ p.p. in $X$.

We shall $u_{\mu}$ the balayaged potential of $u_{\mu}$ to $\omega$ and $\mu^{\prime}$ the balayaged measure of $\mu$ to $\omega$.

Beurling and Deny's Refinement Theorem ${ }^{101}$. For each $u$ in $D$, there exists a function $u^{*}$ defined p.p. in $X$ satisfying the following conditions:

(R. 1) $u^{*}(x)=u(x)$ p.p. in $X$ and $u^{*}(x)$ vanishes outside some o-compact set.

(R. 2) $u^{*}$ is measurable with respect to the measure $\mu$ associated with any pure potential $u_{\mu}$ and

$$
\left(u, u_{\mu}\right)=\int u^{*} d \mu
$$

We shall call $u^{*}$ the refinement of $u$.

Beurling and Deny's Representation Theorem ${ }^{11)}$. For a Dirichlet space $D$, there exist a positive measure $\nu$ in $X$, a positive Hermitian form $N(f, g)$ on $C_{K} \cap D$ and a positive symmetric measure $\sigma$ in $X \times X-\delta$ ( $\delta$ is the diagonal set of $X \times X$ ) such that

$$
(f, g)=\int f \bar{g} d \nu+N(f, g)+\iint(f(x)-f(y))(\bar{g}(x)-\bar{g}(y)) d \sigma(x, y)
$$

for any $f, g$ in $C_{K} \cap D$. Here $N(f, g)$ has the following local character: if $g$ is constant in some neighborhood of the support $S_{f}$ of $f$, then $N(f, g)$ vanishes.

We shall call the measure $\sigma$ the singular measure of $D$. With respect to the measure $\nu$, we have the following

Remark. Let $\left(\omega_{\alpha}\right)_{\alpha \in I}$ be an increaing net of relatively compact open sets tending to $X$, and let $\nu_{\alpha}$ be the equilibrium measure of $\omega_{\alpha}$. Then the net $\left(\nu_{\alpha}\right)_{\alpha \in l}$ converges vaguely to $\nu$.

For the proof, see [2], p. 212. We shall call the measure $\nu$ the equilibrium measure of $X$.

10) Cf. [2], p. 210 .

11) Cf. [2], pp. 211-213. 


\section{Some lemmas}

First we consider the following subspaces of $D$. We put, for a non-empty open set $\omega$ in $X$,

$$
\begin{aligned}
& D_{\omega}^{(1)}=\{\overline{u \in D ; @(u) \subset \omega}\}, \\
& D_{\omega}^{(2)}=\left\{\overline{f \in C_{K} \cap D ; S_{f} \subset \omega}\right\},
\end{aligned}
$$

and for a closed set $F$ in $X$,

$$
\begin{aligned}
& D_{F}^{(1)}=\{u \in D ; \Im(u) \subset F\}, \\
& D_{F}^{(3)}=\left\{u \in D ; u^{*}(x)=0 \text { p.pp. on } F\right\}^{12)} .
\end{aligned}
$$

Lemma 1. For any open set $\omega$ in $X, D_{\omega}^{(2)}=D_{\mathscr{G} \omega}^{(3)}$.

Proof. For any $u$ in $D_{w}^{(2)}$, there exists a sequence $\left(u_{n}\right)$ in $C_{K} \cap D_{w}^{(2)}$ such that $u_{n} \rightarrow u$ strongly in $D$ as $n \rightarrow \infty$. Then for any pure potential $u_{\mu}$ such that $S_{\mu} \subset \mathscr{C} \omega$,

$$
\left(u_{n}, u_{\mu}\right)=\int u_{n} d \mu=0
$$

and hence

$$
\left(u, u_{\mu}\right)=\int u^{*} d \mu=0 .
$$

That is, $u^{*}(x)=0$ p.p.p on $\mathscr{C} \omega$. Consequently $D_{\omega}^{(2)} \subset D_{\mathscr{C} \omega .}^{(3)}$.

Conversely, let $u$ be in $D_{\mathscr{G}}^{(1)}$. By Beurling and Deny's theorem ${ }^{13)}$, there exists a sequence $\left(u_{\mu_{n}}\right)$ such that $u_{\mu_{n}}$ is a linear combination of pure potentials, $\mu_{n}$ is supported by $\mathscr{C}_{\omega}$ and $u_{\mu_{n}} \rightarrow u$ strongly in $D$ as $n \rightarrow \infty$. Then for any $v$ in $D_{\mathscr{C}(v)}^{(3)}$,

$$
\left(v, u_{\mu_{n}}\right)=\int v^{*} d \mu_{n}=0
$$

and hence $(v, u)=0$. This means that $D_{\mathscr{G}(v)}^{(3) \perp} \supset D_{\mathscr{G} v}^{(1)}$. Consequently $D_{w}^{(2)} \supset D_{\mathscr{G}^{(w)}}^{(3)}$, since we have $D_{\omega}^{(2) \perp}=D_{\mathscr{E} \omega}^{(1)}$. This completes the proof.

Lemma 2. An element $u$ in $D$ is a pure potential if and only if there exists a sequence $\left(f_{n}\right)$ of positive bounded measurable functions with compact support such that the sequence $\left(u_{f_{n}}\right)$ of pure potentials converges strongly to $u$ as $n \rightarrow \infty$.

12) A property is said to hold p.p.p. on a subset $E$ in $X$ if the property holds $\mu \cdot p \cdot p$. for any pure potential $u_{\mu}$ in $D$ such that $S_{\mu}=E$.

13) Cf. [2], p. 214. 
For the proof, see Lemma 2 in [2] and [5], p. 2.

Lemma 3. For an element $u$ in $D$ and a real valued continuous function $\varphi$, suppose that $u(x) \geq \varphi(x)$ (resp. $u(x) \leq \varphi(x))$ p.p. in an open set $\omega$. Then $u^{*}(x) \geq \varphi(x)$ (resp. $\left.u^{*}(x) \leq \varphi(x)\right)$ p.p.p. in $\omega$.

Proof. Let $u_{\mu}$ be a pure potential such that $S_{\mu} \subset \omega$. It is sufficient to prove that

$$
\left(u, u_{\mu}\right)=\int u^{*} d \mu \geq \int \varphi d \mu
$$

First suppose that $S_{\mu}$ is compact in $\omega$. By Lemma 2, there exist positive bounded measurable functions $f_{n}$ with compact support such that $\left(u_{f_{n}}\right)$ converges strongly to $u_{\mu}$ in $D$. We take another relatively compact open set $\omega_{1}$ such that

$$
S_{\mu} \subset \omega_{1} \subset \bar{\omega}_{1} \subset \omega .
$$

Put $f_{n, 1}=$ the restriction of $f_{n}$ to $\omega_{1}$ and $f_{n, 2}=$ the restriction of $f_{n}$ to $\mathscr{C} \omega_{1}$. Then the sets $\left(u_{f_{n, 1}}\right)$ and $\left(u_{f_{n, 2}}\right)$ are bounded in $D$. Hence we may assume that there exist $u_{1}$ and $u_{2}$ in $D$ such that

$$
u_{f_{n, 1}} \rightarrow u_{1} \text { and } u_{f_{n, 2}} \rightarrow u_{2}
$$

weakly in $D$, respectively. Since the measure $f_{n}$ converges vaguely to $\mu$, the measure $f_{n, 1}$ converges vaguely to $\mu$, so that for any $f$ in $C_{K} \cap D$,

$$
\left(u_{1}, f\right)=\int \bar{f} d \mu=\left(u_{\mu}, f\right) .
$$

By the denseness of $C_{K} \cap D$ in $D, u_{1}=u_{\mu}$ and $u_{2}=0$. Now let $u$ be the function in our lemma. Then by our assumption,

$$
\left(u_{f_{n, 1}}, u\right)=\int u(x) f_{n, 1}(x) d \xi(x) \geq \int \varphi(x) f_{n, 1}(x) d \xi(x),
$$

$\omega_{1}$ being relatively compact, we obtain

$$
\int \varphi(x) f_{n, 1}(x) d \xi(x) \rightarrow \int \varphi d \mu
$$

as $n \rightarrow \infty$. Consequently

$$
\int u^{*} d \mu \geq \int \varphi d \mu
$$

When $S_{\mu}$ is non-compact, the above result implies that for any restriction $\mu_{K}$ 
of $\mu$ to a compact set $K, u^{*}(x) \geq \varphi(x) \mu_{K}-p$. $p$. in $X$. Hence $u^{*}(x) \geq \varphi(x) \mu-p$. $p$. in $X$. This completes the proof.

Let $u_{\mu}$ be a pure potential in $D$ and $\omega$ be an open set in $X$. Put

$$
E_{u_{\mu, \omega}}=\left\{v \in D ; \operatorname{Rev}(x) \geq u_{\mu}(x) \text { p.p. in } \omega\right\} \text {. }
$$

Beurling and Deny [2] showed that the balayaged potential $u_{\mu^{\prime}}$ of $u_{\mu}$ to $\omega$ is a unique element which minimizes the norm in $E_{u_{\mu}, \ldots}$.

Lemma 3. Let Pru be the projection of a pure potential to $D_{w}^{(1)}$. Then $P r u_{\mu}=u_{\mu^{\prime}}$.

Proof. Since $P r u_{\mu}$ is the projection of $u_{\mu}$ to $D_{\omega}^{(1)}$,

$$
\left(u, P r u_{\mu}\right)=\left(u, u_{\mu}\right)
$$

for any $u$ in $D_{\omega}^{(1)}$. Hence $\operatorname{Pr}_{\mu}(x)=u_{\mu}(x) p . p$. in $\omega$. Therefore

$$
P r u_{\mu} \in E_{u_{\mu} \omega} .
$$

Consequently $\left\|u_{\mu^{\prime}}\right\| \leq\left\|P r u_{\mu}\right\|$. On the other hand, by Beurling and Deny's theorem ${ }^{14)}$, there exists a sequence $\left(u_{n}\right)$ of linear combinations of pure petentials such that $\Im\left(u_{n}\right) \subset \omega$ and $\left(u_{n}\right)$ converges strongly to Pr $u_{\mu}$. Similarly as lemma $2, u_{\mu}^{*}(x)=u_{\mu^{\prime}}^{*}(x)$ p.p.p. in $\omega$. Hence

$$
\left(u_{n}, u_{\mu^{\prime}}\right)=\left(u_{n}, u_{\mu}\right) \rightarrow\left(\operatorname{Pr} u_{\mu}, u_{\mu}\right)=\left\|P r u_{\mu}\right\|^{2} .
$$

Therefore

$$
\left\|u_{\mu^{\prime}}\right\|\left\|P r u_{\mu}\right\| \geq\left(\operatorname{Pr} u_{\mu}, u_{\mu^{\prime}}\right)=\left\|P r u_{\mu}\right\|^{2},
$$

i.e., $\left\|u_{\mu}\right\| \geq\left\|P r u_{\mu}\right\|$. Consequently $u_{\mu^{\prime}}=$ Pr $u_{\mu}$. This completes the proof.

Similarly as above, let $u_{\mu}$ be a potential in $D$ such that $u_{|\mu|}$ in $D$. Then

$$
P r u_{\mu}=u_{\mu_{1}^{\prime}}-u_{\mu_{2}^{\prime}}+i\left(u_{\mu_{3}^{\prime}}-u_{\mu_{4}^{\prime}}^{\prime}\right)
$$

where $\mu=\mu_{1}-\mu_{2}+i\left(\mu_{3}-\mu_{4}\right)$ and $\mu_{j}$ is a positive measure for $j=1,2,3,4$.

LEMma 5. Let $u$ and $v$ be positive elements in $D$ such that $S_{u} \cap S_{v}=\emptyset^{151}$ and $S_{u}$ or $S_{v}$ is compact in $X$. Then $(u, v) \leq 0$.

Proof. Assume that $S_{u}$ is compact. There exists two open sets $\omega_{1}$ and $\omega_{2}$

14) Cf. [2], p. 214 .

15) $S_{u}$ is the complement of the greatest open set in all open sets $G$ that $u(x)=0 p$. in $G$. 
such that $S_{u} \subset \omega_{1}, S_{v} \subset \omega_{2}$ and $\bar{\omega}_{1} \cap \bar{\omega}_{2}=\varnothing$. By Lemma 1, there exist two sequences $\left(f_{n}\right)$ and $\left(g_{n}\right)$ of functions in $C_{K} \cap D$ such that

$$
S_{f_{n}} \subset \omega_{1}, S_{g_{n}} \subset \omega_{2}
$$

and the sequences $\left(f_{n}\right)$ and $\left(g_{n}\right)$ converge strongly to $u$ and $v$, respectively. We may assume that $f_{n}(x) \geq 0$ and $g_{n}(x) \geq 0$ in $X$ for each $n$. Because the sequences $\left(T f_{n}\right)$ and $\left(T g_{n}\right)$ converge strongly to $T u=u$ and $T v=v$, respectively, where $T$ is the following normal contraction: $T(z)=\sup (\operatorname{Rez}, 0)$ for each $z$ in $๘$. By Beurling and Deny's Representation Theorem,

$$
\begin{aligned}
\left(f_{n}, g_{n}\right) & =\iint\left(f_{n}(x)-f_{n}(y)\right)\left(g_{n}(y)\right) d \sigma(x, y) \\
& =-2 \iint f_{n}(x) g_{n}(y) d_{\sigma}(x, y) \leq 0 .
\end{aligned}
$$

Consequently

$$
(u, v)=\lim _{n \rightarrow \infty}\left(f_{n}, g_{n}\right) \leq 0 .
$$

This completes the proof.

Definition 5. Let $A$ be an arbitrary symmetric set in the product space $X \times X$. The projection of the set $(\{x\} \times X) \cap A$ to $X$ is called the $x$-section of $A$ and denoted by $A_{x}$.

Let $F$ be a symmetric closed set in $X \times X$. Put

$$
\tilde{F}=\left\{f(x) g(y) ; f, g \in C_{K}^{+} \cap D, S_{f} \subset \mathscr{C} F_{s_{g}} \text { and } S_{g} \subset \mathscr{C} F_{s_{j}}\right\},
$$

where

$$
F_{s_{p}}=U\left\{F_{x} ; x \in S_{g}\right\} .
$$

Let $C_{K}(\mathscr{C} F)$ be the space of complex-valued continuous functions in $\mathscr{C} F$ with compact support provided with the topology of uniform convergence.

LeMma 6. $\tilde{\mho}$ is total ${ }^{16)}$ in $C_{K}(\mathscr{C F})$.

Proof. It is sufficient to prove that for each point in $\mathscr{C} F$, there exists a base of open neighborhoods of the point satisfying the following condition. For any couple of $\omega_{1}$ and $\omega_{2}$ of the base of open neighboods with

$$
\omega_{1} \subset \bar{\omega}_{1} \subset \omega_{2}
$$

16) This means that the space of linear combinations in $\mathfrak{F}$ is dense in $C_{K}(\mathscr{C} F)$. 
there exists a function $f(x) g(y)$ in $\mathfrak{F}$ such that $f(x) g(y)=1$ in $\omega_{1}$ and $f(x) g(y)=0$ on $\mathscr{C} \omega_{2}$. We put

$$
\Re(x, y)=\{U(x) \times U(y) ; U(x) \times U(y) \subset \mathscr{C} F\},
$$

where $U(x)$ and $U(x)$ are open neighborhoods of $x$ and $y$, respectively. Then $\mathfrak{R}(x, y)$ is a base of open neighborhoods of $(x, y) \in \mathscr{C} F$. For a couple of $U\left(x_{0}\right) \times U\left(y_{0}\right)$ and $V\left(x_{0}\right) \times V\left(y_{0}\right)$ in $\Re\left(x_{0}, y_{0}\right)$ with

$$
\overline{U\left(x_{0}\right)} \subset V\left(x_{0}\right) \text { and } \overline{U\left(y_{0}\right)} \subset V\left(y_{0}\right),
$$

we construct a function $f(x) g(y)$ as above. Since $C_{K}^{+} \cap D$ is dense in $C_{K}^{+1 i}$, there exists functions $f_{1}$ and $g_{1}$ in $C_{K}^{+} \cap D$ such that $f_{1}(x) \geq 0$ in $U\left(x_{0}\right), f_{1}(x)=0$ on $\mathscr{C} V\left(x_{0}\right), g_{1}(x) \geq 1$ in $U\left(y_{0}\right)$ and $g_{1}(x)=0$ on $\mathscr{C} V\left(y_{0}\right)$. Let $T$ be the projection of the complex plane to the closed interval $[0,1]$. Then $T$ is a normal contraction. Put

$$
f(x)=T f_{1}(x) \text { and } g(x)=T g_{1}(x) .
$$

Then $f$ and $g$ is contained in $C_{K}^{+} \cap D$,

$$
f(x) g(y)=1
$$

in $U\left(x_{0}\right) \times U\left(y_{0}\right)$ and

$$
f(x) g(y)=0
$$

on $\mathscr{C}\left(V\left(x_{0}\right) \times V\left(y_{0}\right)\right)$. Next we shall prove that

$$
S_{f} \subset \mathscr{C} F_{s_{g}} \text { and } S_{g} \subset \mathscr{C} F_{s_{f}} .
$$

It is sufficient to show that

$$
F_{V\left(x_{v}\right)} \cap V\left(y_{0}\right)=\varnothing \text { and } F_{V\left(y_{0}\right)} \cap V\left(x_{0}\right)=\varnothing \text {. }
$$

Suppose that $F_{V\left(x_{0}\right)} \cap V\left(y_{0}\right) \neq \varnothing$. Then there exists a point $x_{1}$ in $V\left(x_{0}\right)$ such that $F_{x_{1}} \cap V\left(y_{0}\right) \neq \varnothing$. Let $x_{2}$ be a point of $F_{x_{1}} \cap V\left(y_{0}\right)$. Since

$$
\left(x_{1}, x_{2}\right) \in\left\{x_{1}\right\} \times F_{x_{1}} \subset F
$$

and

$$
\begin{aligned}
& \left(x_{1}, x_{2}\right) \in V\left(x_{0}\right) \times V\left(y_{0}\right), \\
& V\left(x_{0}\right) \times V\left(y_{0}\right) \cap F \neq \varnothing .
\end{aligned}
$$

17) Since $C_{K} \cap D$ is dense in $C_{K}$, for a function $f$ in $C_{K}^{+}$, there exists a sequence $\left(f_{n}\right)$ in $C_{K} \cap D$ such that it converges to $f$ in $C_{K}$. Since $f$ is positive, $\left(f_{n}^{+}\right)$converges to $f$ in $C_{K} . f_{n}^{+}$is contained in $D$. 
This contradicts our assumption and hence $F_{V\left(x_{0}\right)} \cap V\left(y_{0}\right)=\varnothing$. Similarly $F_{r^{\prime}\left(y_{0}\right)} \cap V\left(x_{0}\right)=\emptyset$. This completes the proof.

\section{First main theorems}

First we give the following definition.

Definition 6. A symmetric closed set $F$ in $X \times X$ is said to be regular if the mapping: $x \in X \rightarrow F_{x}$ is continuous, that is, for any open set $\omega$ containg $F_{x}$, there exists a neighborhood $U(x)$ of $x$ such that $F_{y} \subset \omega$ for any $y$ in $U(x)$.

One of our main theorems is the following

Theorem 1. Let $D$ be a Dirichlet space with respect to $X$ and $\xi$, and let $F$ be a regular closed set in $X \times X$ such that for any $x$ in $X$, the $x$-section $F_{x}$ is compact and $F$ contain the diagonal set $\delta$ of $X \times X$. Then the following two conitions are equivalent.

(I. 1) The singular measure of $D$ is supported by $F$.

(I. 2) For a function $u$ in $D$ and a point $x_{0}$ in $X$, if there exists an open set $\omega$ such that $\omega \supset F_{x_{0}}$ and $u(x)=0$ p.p. in $\omega$, then $x_{0} \notin \varsigma(u)$.

Proof. First we prove the implication (I. 1) $\Rightarrow($ I. 2). We can take another open set $\omega_{1}$ such that

$$
F_{x_{0}} \subset \omega_{1} \subset \bar{\omega}_{1} \subset \omega,
$$

because $F_{x_{0}}$ is compact. By Lemmas 1 and 2, there exists a sequence $\left(u_{n}\right)$ of $C_{K} \cap D$ such that $S_{u_{n}} \subset \mathscr{C} \bar{\omega}_{1}$ and $\left(u_{n}\right)$ converges strongly to $u$ in $D$ as $n \rightarrow \infty$. Since $F$ is regular, there exists an open neighborhood $V\left(x_{0}\right)$ of $x_{0}$ such that $F_{y} \subset \omega_{1}$ for any $y$ in $V\left(x_{0}\right)$. For any $f$ in $C_{K} \cap D$ with support in $V\left(x_{0}\right)$,

$$
\begin{aligned}
\left(u_{n}, f\right) & =\iint\left(u_{n}(x)-u_{n}(y)\right)(\bar{f}(x)-\bar{f}(y)) d \sigma(x, y) \\
& =-2 \iint u_{n}(x) \bar{f}(y) d \sigma(x, y)=0 .
\end{aligned}
$$

Here we used the symmetricity of $\sigma$. By this equality, it follows that $\mathfrak{E}\left(u_{n}\right) \subset \mathscr{C} V\left(x_{0}\right)$ for any $n$. Consequently

$$
\Subset(u) \subset \mathscr{C} V\left(x_{0}\right) \text {. }
$$

Next we prove the converse. Suppose that $F \nsubseteq S o$. By Lemma 5, there exists an element $f(x) g(y)$ in $\tilde{f}$ such that 


$$
\iint f(x) g(y) d \sigma(x, y)>0
$$

On the other hand by our assumption,

$$
F_{S_{g}} \subset \mathscr{C} S_{f} \text {, so that } S_{g} \subset \mathscr{C} \cong(f) .
$$

Therefore $(f, g)$ vanishes. This contradicts our assumption and the proof is complete.

Applying the above theorem, we obtain the following

Theorem 2. Let $D$ and $F$ be same as in Theorem 1. Then the condition (I.1) is satisfied in D if and only if the following condition (II. 1) is satisfied in D.

(II. 1) For any pure potential $u_{\mu}$ in $D$ and any closed neighborhood $\omega$ of $S_{\mu}$, let $u_{\mu}$ be the balayaged potential of $u_{\mu}$ to $\mathscr{C} \omega$. Then

$$
S_{\mu}, \subset F \omega \cap \overline{\mathscr{C} \omega}
$$

Proof. By Theorem 1, it is sufficient to prove that the condition (I. 2) and (II. 1) are equivalent. First we prove the implication (I. 2) $\Rightarrow($ II. 1). For a pure potential $u_{\mu}$ and a closed neighborhood $\omega$ of $S_{\mu}$, let $u_{\mu^{\prime}}$ be the balayaged potential of $u_{\mu}$ to $\mathscr{C} \omega$. It is sufficient to prove

$$
\int f(x) d \mu^{\prime}=0
$$

for any $f$ in $C_{K}^{+} \cap D$ with support in $\mathscr{C} F \omega$. Since $f(x)=0$ in some neighborhood of $F \omega, \Subset(f) \subset \mathscr{C} \omega$ by the condition (I. 2).

(1) The case that $S_{\mu}$ and $\omega$ are compact. We can take an open set $\omega_{1}$ in $X$ such that

$$
\bar{\omega} \subset \omega_{1} \subset \bar{\omega}_{1} \subset \mathscr{C} \Im(f) .
$$

By Lemma 1, there exists a sequence $\left(u_{n}\right)$ in $C_{K} \cap D$ supported by $\omega_{1}$ such that $\left(u_{n}\right)$ converges strongly to $u_{\mu}-u_{\mu}$ in $D$, because

$$
u_{\mu}^{*}(x)-u_{\mu^{\prime}}^{*}(x)=0
$$

p.p.p. on $\mathscr{C} \omega_{1}$. Obviously $\left(u_{n}, f\right)=0$, so that

$$
\left(u_{\mu}-u_{\mu^{\prime}}, f\right)=0 \text {. }
$$

That is, 


$$
\begin{aligned}
\left(u_{\mu}-u_{\mu^{\prime}}, f\right) & =\int f(x) d \mu-\int f(x) d \mu^{\prime} \\
& =-\int f(x) d \mu^{\prime}=0 .
\end{aligned}
$$

(2) The general case. Let $K$ be a compact set in $X$. Let $\mu_{K}$ be the restriction of $\mu$ to $K$. We take an increasing net $\left(\omega_{\alpha}\right)_{\alpha \in i}$ of compact neighborhoods of $S_{\mu_{K}}$ such that $\left(\omega_{\alpha}\right)_{\alpha \in I} \nearrow \omega$. By the case (1),

$$
S_{\mu_{K, \alpha}^{\prime}} \subset F_{\omega_{\alpha}} \subset F_{\omega}
$$

where $\mu_{K, \alpha}^{\prime}$ is the balayaged measure of $\mu_{K}$ to $\mathscr{C} \omega_{\alpha}$. Let $\mu_{K}^{\prime}$ be the balayaged measure to $\mathscr{C} \omega$. Since the net $\left(u_{\mu_{K, \alpha}^{\prime}}\right)$ converges strongly to $u_{\mu_{K}^{\prime}}$ in $D$,

$$
S_{\mu_{K}^{\prime}} \subset F_{\omega} \cap \overline{\mathscr{C} \omega} \text {. }
$$

Next making $K$ tend to $X$, we obtain that $\left(u_{\mu_{K}^{\prime}}\right)$ converges strongly to $u_{\mu^{\prime}}$ in D. Consequently

$$
S_{\mu}, \subset F_{\omega} \cap \overline{\mathscr{C} \omega}
$$

Now we prove the implication (II. 1$) \Longrightarrow($ I. 2). For a function $u$ in $D$, suppose that there exists a point $x_{0}$ such that $u(x)=0 p . p$. in some open set $\omega$ containing $F_{x_{0}}$. By the regularity of $F$, there exists an open neighborhood $V\left(x_{0}\right)$ such that $F_{y} \subset \omega$ for each $y$ in $V\left(x_{0}\right)$. We take another neighborhood $V_{1}\left(x_{0}\right)$ such that

$$
V_{1}\left(x_{0}\right) \subset \overline{V_{1}\left(x_{0}\right)} \subset V\left(x_{0}\right) .
$$

For a pure potential $u_{\mu}$, let $u^{\prime}$ be the projection of $u_{\mu}$ to $D_{\mathscr{C} V_{1}\left(x_{0}\right)}^{(1)}$. If $\mu$ is supported by $V_{1}\left(x_{0}\right), \Im\left(u^{\prime}\right)$ is contained in $\omega$ by Lemma 3 . Hence

$$
\left(u, u_{\mu}-u^{\prime}\right)=0
$$

because we can take a sequence $\left(u_{n}\right)$ in $C_{K} \cap D$ such that it converges strongly to $u$ in $D$ and

$$
S_{u_{n}} \subset \mathscr{C}\left(\Im\left(u^{\prime}\right) \cup V_{1}\left(x_{0}\right)\right) .
$$

Since the space of linear combinations of such elements $u_{\mu}-u^{\prime}$ is dense in $D_{r_{1}\left(x_{0}\right)}^{(2)},(u, v)=0$ for any $v$ in $D_{v_{1}\left(x_{0}\right)}^{(2)}$. Therefore $x_{0} \notin \Im(u)$. This completes the proof.

Especially when $F=\delta$, we obtain the following. 
TheOREM $2^{\prime}$. Let $D$ be the same as in Theorem 1. The following two conditions are equivalent.

(II'. 1) For any pure potential $u_{\mu}$ in $D$ and any neighborhood $\omega$ of $S_{\mu}$, let $u_{\mu}$ be the balayaged potential of $u_{\mu}$ to $\mathscr{C} \omega$. Then

$$
S_{\mu}, \subset \partial \omega .
$$

(II'. 2) For any $f, g$ in $C_{K} \cap D$,

$$
(f, g)=\int f \bar{g} d \nu+N(f, g) .
$$

Now we consider the relation between the supports of condensor measures and that of the singular measure of $D$.

Theorem 3. Let $D$ and $F$ be the same as in Theorem 1. Let $\nu$ be the equilibrium measure of $X$. Then the following conditions are equivalent:

(III. 1) The singular measure $\sigma$ of $D$ is supported by $F$.

(III. 2) For any couple of open sets $\omega_{1}$ and $\omega_{0}$ with disjoint closures, $\omega_{1}$ being relatively compact, let $u_{\mu}$ be the condensor potential with respect to $\omega_{1}$ and $\omega_{0}$. Then

$$
S_{\mu+} \subset\left(F_{\mathscr{C}_{\omega_{1}}} \cup S_{\nu}\right) \cap \bar{\omega}_{1}
$$

and

$$
S_{\mu}-\subset F_{\mathscr{C} \omega_{0}} \cap \bar{\omega}_{0} .
$$

Proof. First we shall prove the implication (III. 1) $\Longrightarrow$ (III. 2). By Theorem 1 , it is obvious that

$$
S_{\mu-} \subset F_{\mathscr{C} \omega_{0}}
$$

That is,

$$
S_{\mu}-\subset F_{\mathscr{C}_{\omega_{0}}} \cap \bar{\omega}_{0}
$$

On the other hand, for a function $f$ with support in $\mathscr{C}\left(F_{\mathscr{C}_{v_{1}}} \cup S_{\nu}\right) \cap \omega_{1}$, we prove that $\left(u_{\mu}, f\right)=0$. It is obvious that the element $u_{\mu}$ is the element whose norm is minimum in the set $\overline{E_{0,1}}$, where

$$
E_{0,1}=\left\{f \in C_{K} \cap D ; f(x) \geq 1 \text { in } \omega_{1} \text { and } f(x) \leq 0 \text { in } \omega_{0}\right\} .
$$

Hence there exists a sequence $\left(f_{n}^{\prime}\right)$ in $E_{0,1}$ such that $\left(f_{n}^{\prime}\right)$ converges strongly to $u_{\mu}$ in $D$. Put

$$
f_{n}(x)=T f_{n}^{\prime}(x)
$$


where $T$ is the projection of $\Subset$ to the closed interval $[0,1]$. Then $f_{n}$ is contained in $E_{0,1}$ and the sequence $\left(f_{n}\right)$ converges strongly to $T u_{\mu}=u_{\mu}$ in $D$. We have

$$
\begin{aligned}
\left(f, f_{n}\right) & =\int f \bar{f}_{n} d \nu+N\left(f, f_{n}\right)+\iint(f(x)-f(y))\left(\bar{f}_{n}(x)-\bar{f}_{n}(y)\right) d \sigma(x, y) \\
& =\iint(f(x)-f(y))\left(f_{n}(x)-f_{n}(y)\right) d \sigma(x, y) \\
& =2 \iint f(x)\left(f_{n}(x)-f_{n}(y)\right) d \sigma(x, y) \\
& =2 \iint f(x)\left(1-f_{n}(y)\right) d \sigma(x, y)=0,
\end{aligned}
$$

because the support of the function $f(x)\left(1-f_{n}(y)\right)$ is contained in $\mathscr{C} F_{\mathscr{C} \omega_{1}} \times \mathscr{C} \omega_{1}$, and

$$
\left\{\mathscr{C} F_{\mathscr{C} \omega_{1}} \times \mathscr{C} \omega_{1}\right\} \cap F=\varnothing .
$$

Therefore

$$
S_{\mu+} \subset \mathscr{C}\left(F_{\mathscr{C}_{\omega_{1}}} \cup S_{\nu}\right) \cap \bar{\omega}_{1}
$$

Next we prove the implication (III. 2) $\Rightarrow$ (III. 1). For a function $f$ in $C_{K} \cap D$, suppose that there exists a point $x_{0}$ such that $f(x)=0$ in some open set $\omega$ containing $F_{x_{0}}$. By the regularity of $F$, there exists an open neighborhood $V\left(x_{0}\right)$ of $x_{0}$ such that $F_{y} \subset \omega$ for each $y$ in $V\left(x_{0}\right)$. We take a couple of open neighborhoods $U_{1}\left(x_{0}\right)$ and $U_{0}\left(x_{0}\right)$ of $x_{0}$ such that

$$
\overline{U_{1}\left(x_{0}\right)} \subset U_{0}\left(x_{0}\right) \subset \overline{U_{0}\left(x_{0}\right)} \subset V\left(x_{0}\right) .
$$

Let $u_{\mu}$ be the condensor potential with respect to $U_{1}\left(x_{0}\right)$ and $\mathscr{C} \overline{U_{0}\left(x_{0}\right)}$. By our assumption,

$$
\left(u_{\mu}, f\right)=0 .
$$

For any $g$ in $C_{K}^{+} \cap D$ with support in $V\left(x_{0}\right)$, as we may assume that

$$
U_{1}\left(x_{0}\right) \supset S_{g},
$$

there exists a positive constant $M$ such that

$$
g(x) \leq M u_{\mu}(x)
$$

p.p. in $X$. By Lemma 5 ,

$$
0=M\left(u_{\mu}, f\right) \leq(g, f) \leq 0 .
$$

Hence $(g, f)=0$, i.e., $V\left(x_{0}\right) \subset \mathscr{C} \Subset(f)$. Using the same method as in the proof of Theorem 2, we can prove that the condition (I. 2) is satisfied. This completes 
the proof.

Similarly as Theorem $2^{\prime}$, putting $F=\delta$, we obtain the following

Theorem 3'. Let $D$ be the same as in Theorem 1 . Then the following two conditions are equivalent:

(III'. 1) For any $f, g$ in $C_{K} \cap D$,

$$
(f, g)=N(f, g) \text {. }
$$

(III'. 2) For any couple of open sets $\omega_{1}$ and $\omega_{0}$ with disjoint closures, $\omega_{1}$ being relatively ompact, let $u_{\mu}$ be the condensor potential with respect to $\omega_{1}$ and $\omega_{0}$. Then

$$
S_{\mu} \subset \partial \omega_{1} \text { and } S_{\mu}-\subset \partial \omega_{0} .
$$

In this theorem, we remark that if the condition (III'. 2) is satisfied, the equilibribrium measure of $X$ vanishes.

\section{Preliminaries on special Dirichlet spaces and some lemmas}

First we define a negative definite function in Beurling's sense.

Definition 7. ${ }^{18)} \quad A$ complex-valued continuous function $\lambda(x)$ on a locally compact abelian group $X$ is called a negative definite function if the following form

$$
\sum_{i, j=1}^{n}\left\{\lambda\left(x_{i}\right)+\overline{\lambda\left(x_{j}\right)}-\lambda\left(x_{i}-x_{j}\right)\right\} \rho_{i} \overline{\rho_{j}}
$$

is non-negative for each set of $n$ points $x_{1}, x_{2}, \ldots, x_{n}$ in $X$ and $n$ complex numbers $\rho_{1}, \rho_{2}, \ldots, \rho_{n}$.

With respect to a negative definite function, the following result is $\mathrm{known}^{19}$. Let $\lambda(x)$ be a negative definite function on $X$. Then $(\lambda(x))^{-1}$ is a measure of positive type if it is locally summable for the Haar measure.

Definition 8. ${ }^{20)} \quad A$ Dirichlet space $D=D(X, \xi)$ is said to be special if $X$ is a locally compact abelian group and $\xi$ is the Haar measure on $X$, the following condition being satisfied:

d) If $U_{x} u$ is the function obtained from $u$ in $D$ by the translation $x \in X$ (i.e., $\left.U_{x} u(y)=u(y-x)\right)$, then $U_{x} u$ is in $D$ and $\left\|U_{x} u\right\|=\|u\|$.

18) Cf. [2], p. 210, and [4], pp. 8-9.

19) Cf. [4], p. 8.

20) Cf. [2], p. 215, and [4], p. 9 . 
Beurling and Deny [2] obtained the following important theorem.

Beurling and Deny's 2nd Representation Theorem²1). For a special Dirichlet space $D$ on $X$, there exists a real-valued negative definite function $\lambda(\hat{x})$ on the dual group $\hat{X}$ of $X$ such that $(\lambda(\hat{x}))^{-1}$ is locally summable and

$\left(R^{\prime} .1\right)$

$$
\|u\|^{2}=\int|\hat{u}(\hat{x})|^{2} \lambda(\hat{x}) d \hat{x}
$$

for any $u$ in $C_{K} \cap D$, where $\hat{u}$ is the Fourier transform of $u$.

Conversely, such a negative definite function $\lambda(x)$ on $\hat{X}$ defines by means of $\left(\mathrm{R}^{\prime} .1\right)$, a special Dirichlet space on $X$.

Hereafter in this section, we assume that $X$ is a locally compact abelian group. By the above-mentioned result with respect to a negative definite function and by Bochner's theorem, there exists, for each special Dirichlet space on $X$, a positive measure $\kappa$ on $X$ such that the generalized Fourier transform is equal to $(\lambda(x))^{-1}$. This positive measure is called the associated convolution kernel (or simply, kernel) of $D$.

Lemma 7. Let $D$ be a special Dirichlet space on $X$. For each potential $u_{\mu}$ in $D$ such that $\int d|\mu|<+\infty$, it holds that

$$
u_{\mu}=\kappa * \mu \text {. }
$$

Proof. By Beurling and Deny's 2nd Representation Theorem, the transformation $u \rightarrow \hat{u}$ of $C_{K} \cap D$ into $L^{2}(\lambda)$ is isometric. Since $C_{K} \cap D$ is dense in $D$, there exists an element $\tilde{u}_{\mu}(\hat{x})$ in $L^{2}(\lambda)$ such that

$$
\left\|u_{\mu}\right\|^{2}=\int\left|\tilde{u}_{\mu}(\hat{x})\right|^{2} \lambda(\hat{x}) d \hat{x} .
$$

By the above equality and Parseval's formula,

$$
\begin{aligned}
& \int \tilde{u}_{\mu}(\hat{x}) \overline{\hat{\varphi}}(\hat{x}) \lambda(\hat{x}) d \hat{x}=\left(u_{\mu}, \varphi\right) \\
= & \int \bar{\varphi} d \mu=\int \hat{\mu}(\hat{x}) \overline{\hat{\varphi}}(\hat{x}) d \hat{x} .
\end{aligned}
$$

for any $\varphi$ in $C_{K} \cap D$. Since the set $\left\{\hat{\varphi} ; \varphi \in C_{K} \cap D\right\}$ is dense in $C_{K}(\dot{\hat{X}})$, ${ }^{22)}$

21) Cf. [4], pp. 9-14, and [2], p. 215.

22) Cf. [4], p. 10. 


$$
\tilde{u}_{\mu}(\hat{x})=\frac{\hat{\mu}(\hat{x})}{\lambda(\hat{x})}
$$

p.p. in $\hat{X}$. On the other hand $\kappa * \mu$ exists and

$$
\hat{\kappa * \mu}(\hat{x})=\frac{\hat{\mu}(\hat{x})}{\lambda(\hat{x})}
$$

p.p. in $\hat{X}$, since the total mass of $|\mu|$ is finite. Hence

$$
\tilde{u}_{\mu}(\hat{x})=\widehat{\kappa * \mu}(\hat{x})
$$

p.p. in $\hat{X}$. By Parseval's formula,

$$
\begin{aligned}
& \int \bar{\varphi}(x) u_{\mu}(x) d x=\left(u_{\mu}, u_{p}\right)=\int \tilde{u}_{\mu}(\hat{x}) \overline{\widetilde{u}}_{p \vartheta}(\hat{x}) \lambda(\hat{x}) d \hat{x} \\
= & \int \kappa * \mu(\hat{x}) \overline{\hat{\varphi}}(\hat{x}) d \hat{x}=\int \bar{\varphi}(x) d(\kappa * \mu)
\end{aligned}
$$

for any $\varphi$ in $C_{K}$. Consequently $u_{\mu}=\kappa * \mu$. This completes the proof.

Lemma 8. Let $D$ be a special Dirichlet space on $X$. Put

$$
P_{0}=\left\{u_{\mu} \in C_{K} \cap D ; \int d|\mu|<+\infty\right\} \text {. }
$$

Then $P_{0}$ is total in $C_{K}$.

Proof. Similarly as the proof of Lemma 6 , it is sufficient to prove that for any couple of open sets $\omega_{1}$ and $\omega_{2}$ with $\bar{\omega}_{1} \subset \omega_{2}, \omega_{1}$ being relatively compact, there exists an element $u_{\mu}$ in $P_{0}$ such that

$$
\begin{aligned}
& u_{\mu}(x)=1 \text { in } \omega_{1}, \\
& S_{u_{\mu}} \subset \omega_{2} .
\end{aligned}
$$

We take other relatively compact open sets $\omega_{1}^{\prime}$ and $\omega_{2}^{\prime}$ such that

$$
\bar{\omega}_{1} \subset \omega_{1}^{\prime} \subset \bar{\omega}_{1}^{\prime} \subset \omega_{2}^{\prime} \subset \bar{\omega}_{2}^{\prime} \subset \omega_{2} .
$$

Let $u_{\nu}$ be the condensor potential associated with $\omega_{1}^{\prime}$ and $\mathscr{C} \bar{\omega}_{2}^{\prime}$. Then there exists a function $\varphi$ in $C_{K}$ such that

$$
u_{\nu} * \varphi=1 \text { in } \omega_{1} \text { and } S_{u_{\nu} * \vartheta} \subset \omega_{2} .
$$

It is known that $u_{\downarrow} * \varphi$ is contained in $D .^{23)}$ By Lemma 7,

23) Cf. [4], p. 7 . 


$$
u_{\nu} * \varphi=u_{\nu * \varphi}
$$

since the total mass of $|\nu|$ is finite, it holds that

$$
\int|\nu * \varphi(x)| d x<+\infty
$$

Hence $u_{\nu} * \varphi$ belongs to $P_{0}$. This completes the proof.

Lemma 9 (Unicity Theorem). Let $D$ be a special Dirichlet space on $X, \kappa$ be the kernel of $D$ and let $\mu_{1}, \mu_{2}$ be measures on $X$ such that

$$
\int d\left|\mu_{1}\right|<+\infty \text { and } \int d\left|\mu_{2}\right|<+\infty .
$$

If $\kappa * \mu_{1}=\kappa * \mu_{2}$, then $\mu_{1}=\mu_{2}$.

Proof. By Lemma 8, it is sufficient to prove the equality

$$
\int \mu_{\mu}(x) d \mu_{1}(x)=\int u_{\mu}(x) d \mu_{2}(x)
$$

for any $u_{\mu}$ in $P_{0}$. Since $\kappa$ is symmetric,

$$
\begin{aligned}
& \int u_{\mu}(x) d \mu_{1}(x)=\kappa * \mu * \check{\mu}_{1}(0)=\left(\kappa * \mu_{1}\right) * \check{\mu}(0) \\
&=\left(\kappa * \mu_{2}\right) * \check{\mu}(0)=\kappa * \mu * \check{\mu}_{2}(0)=\int u_{\mu}(x) d \mu_{2}(x) .
\end{aligned}
$$

This completes the proof.

Remark. The above result is evident, when both $u_{\mu_{1}}$ and $u_{\mu_{2}}$ are in $D$.

Lemma 10. Let $D$ be a special Dirichlet space on $X$ and let $k$ be the kernel of $D$. For each $x$ in $X$ and each closed neighborhood $\omega$ of $x$, there exists a positive measure $\varepsilon_{x}^{\prime}$ such that

$$
\begin{aligned}
& \varepsilon_{x}^{\prime} \text { is supported by } \overline{\mathscr{C} \omega} \text { and } \int d \varepsilon_{x}^{\prime} \leq 1, \\
& \kappa * \varepsilon_{x}=\kappa * \varepsilon_{x}^{\prime} \text { as a measure in } \mathscr{C} \omega, \\
& \kappa * \varepsilon_{x} \geq \kappa * \varepsilon_{x}^{\prime} .
\end{aligned}
$$

Here $\varepsilon_{x}$ is a unit measure at $x$.

Proof. Without loss of generality we may assume that $x=0$. There exists a sequence $\left(\varphi_{n}\right)$ in $C_{K}$ such that

$$
\varphi_{n}(x) \geq 0, \int \varphi_{n}(x) d x=1
$$


and it converges vaguely to $\varepsilon\left(=\varepsilon_{0}\right)$ as $n \rightarrow \infty$. By Beurling and Deny's Balayage Theorem,- there exists the balayaged measure $\varphi_{n}^{\prime}$ of the measure $\varphi_{n}$ to $\mathscr{C} \omega$. For each $n$, the total mass of $\varphi_{n}^{\prime}$ being less than or equal to 1 , the set $\left\{\varphi_{n}^{\prime}\right\}$ is bounded in the vague topology. Hence we may assume that there exists a positive measure $\varepsilon^{\prime}$ to which $\left(\varphi_{n}^{\prime}\right)$ tends vaguely. On the other hand since

$$
\kappa * \varphi_{n}^{\prime} \leq \kappa * \varphi_{n}
$$

and the sequence $\left(\kappa * \varphi_{n}\right)$ converges vaguenely to $\kappa$, the set $\left\{\kappa * \varphi_{n}^{\prime}\right\}$ is bounded in the vague topology. We may assume that there exists a positive measure $\eta$ to which $\left(\kappa * \varphi_{n}^{\prime}\right)$ converges vaguely. Hereafter we prove that $\eta=\kappa * \varepsilon^{\prime}$. Since the total mass of $\varepsilon^{\prime}$ is finite $(\leq 1), \kappa * \varepsilon^{\prime}$ exists. It holds that

$$
\kappa * \varphi * \bar{\varphi}(x)=\left(u_{p}, U_{x} u_{p}\right)=\int \frac{|\check{\varphi}(\hat{x})|^{2}}{\lambda(\hat{x})}(\hat{x}, x) d \hat{x}
$$

for any $\varphi$ in $C_{K}$, where $(\hat{x}, x)$ is a character of $X$. The function $\lambda^{-1}|\hat{\varphi}(\hat{x})|^{2}$ being integrable, $\kappa * \varphi * \bar{\varphi}(x)$ is 0 at infinity for each $\varphi$ in $C_{K}$. Therefore

$$
\int \varphi * \overline{\breve{\varphi}}(x) d\left(\kappa * \varphi_{n}^{\prime}\right)=\int \kappa * \varphi * \overline{\breve{\varphi}}(x) d \varphi_{n}^{\prime} \rightarrow \int \kappa * \varphi * \overline{\breve{\varphi}}(x) d \varepsilon^{\prime},
$$

because the boundedness of the total masses and the vague convergence to $\varepsilon^{\prime}$ of $\left(\varphi_{n}^{\prime}\right)$ implies the convergence

$$
\lim _{n \rightarrow \infty} \int f(x) d \varphi_{n}^{\prime}=\int f(x) d \varepsilon^{\prime}
$$

for any complex-valued continuous function $f$ with 0 at infinity. Hence

$$
\int \varphi * \bar{\varphi}(x) d\left(\kappa * \varepsilon^{\prime}\right)=\int \varphi * \bar{\varphi}(x) d \eta
$$

The set $\left\{U_{x} \varphi * \bar{\varphi} ; \varphi \in C_{K}, x \in X\right\}$ being total in $C_{k}$, we have $\kappa * \varepsilon^{\prime}=\eta$, which prove the conditions $\left(\mathrm{B}^{\prime} .1\right),\left(\mathrm{B}^{\prime} .2\right)$ and $\left(\mathrm{B}^{\prime} .3\right)$.

Remark. Similarly as above, for a positive measure $\mu$ with finite total mass and for an open set $\omega$, there exists a positive measure $\mu^{\prime}$ such that

$$
\mu^{\prime} \text { is supported by } \bar{\omega} \text { and } \int d \mu^{\prime} \leq \int d \mu \text {, }
$$$$
\kappa * \mu \iota^{\prime}=\kappa * \mu \text { as a measure in } \omega \text {, }
$$$$
\kappa * \mu \ell^{\prime} \leq \kappa * \mu \text {. }
$$ 
In this remark, the total mass of $\mu$ being finite, we obtain that the convolution $\kappa * \mu$ exists.

We call the measure $\mu^{\prime}$ the balayaged measure of $\mu$ to $\omega$, as same as in Beurling and Deny's Balayage Theorem. It is an unknown problem if the balayaged measure $\mu$ to $\omega$ is unique when $u_{\mu}$ is not contained in $D$.

\section{Second main theorems}

In this section, we consider a special Dirichlet space on the $n$-dimensional Euclidean space $R^{n}(n \geq 1)$. Let $\lambda(x)$ be a real valued negative definite function in $R^{n}$ such that $\lambda^{-1}$ is locally summable. Then by Levy-Khintchine's theorem, we have the following representation:

$$
\lambda(x)=C+\sum_{i, j=1}^{n} a_{i j} x_{i} x_{j}+\int_{|y|>0}\left(1-e^{2 \pi i x \cdot y}\right) d \sigma_{1}(y),
$$

where $C$ is a non-negative constant, the second term is a positive quardratic form and $\sigma_{1}$ is a positive symmetric measure in $R^{n}-\{0\}$ such that

$$
\int_{|x|<r} d \sigma_{1}<+\infty \text { and } \int_{0<|x|<r}|x|^{2} d \sigma_{1}(x)<+\infty
$$

for any $r>0$.

Similarly as Theorem 2, we obtain the following

Theorem 4. Let $D$ be a special Dirichlet space on $R^{n}$ and let $\lambda(x)$ be a negative definite function associated with $D$. Given a symmetric (with respect to the origin) compact set $K$ containing 0 , the following conditions are equivalent.

(IV. 1) Let $\varepsilon_{r}^{\prime}$ be the balayaged measure of a unit measure $\varepsilon$ at 0 to the outside of a closed ball $\bar{B}(0 ; r)$ with center at 0 and radius $r$. Then

$$
S_{\varepsilon_{r}^{\prime}} \subset(\bar{B}(0 ; r)+K) \cap \mathscr{C} B(0 ; r),{ }^{24)}
$$

where $B(0 ; r)$ is the open ball with center at 0 and of radius $r$.

(IV. 2) $\sigma_{1}=0$ as a measure in $\mathscr{C} K$.

(IV. 3) For each $x$ in $R^{n}$ and each closed neighborhood $\omega$ of $x$, let ${ }^{-} \varepsilon_{x}^{\prime}$ be the balayaged measure of a unite measure $\varepsilon_{x}$ at $x$ to $\mathscr{C} \omega$. Then

24. Let $A$ and $B$ be subsets in $R^{n}$. $A+B$ means the set $\{x+y ; x \in A, y \in B\}$ and $A-B$ means the set $\{x-y ; x \in A, y \in B\}$. 


$$
S_{\varepsilon_{x}^{\prime}} \subset(\omega+K) \cap \overline{\mathscr{C} \omega}
$$

Before the proof, we remark the following result. By Beurling and Deny's 2nd Representation Theorem, for any $f, g$ in $C_{K} \cap D$,

$$
(f, g)=\int \hat{f}(\hat{x}) \overline{\hat{g}}(\hat{x}) \lambda(\hat{x}) d \hat{x} .
$$

By Parseval's formula,

$$
\begin{gathered}
(f, g)=C \int f(x) \overline{\hat{g}}(x) d x+\sum_{i, j=1}^{n} a_{i j} \int \frac{\partial f}{\partial x_{i}} \frac{\overline{\partial g}}{\partial x_{j}} d x \\
+\iint(f(x+y)-f(x))(\bar{g}(x+y)-\bar{g}(x)) d \sigma_{1}(y) d x
\end{gathered}
$$

for any $f, g$ in $C_{K}^{2} \cap D$. Therefore we obtain that the singular measure $\sigma$ of $D$ is as follows:

$$
\int f(x) g(y) d \sigma(x, y)=\iint f(x+y) g(x) d \sigma_{1}(y) d x
$$

for any $f, g$ in $C_{K}$ such that

$$
S_{f} \cap S_{g}=\varnothing .
$$

Proof of Theorem 4. First we prove the implication (IV.1) $\Rightarrow($ IV.2). Let $\kappa$ be the kernal of $D$ and $\sigma_{m}^{(1)}$ be the restriction of $\sigma_{1}$ to $\mathscr{C} B\left(0 ; m^{-1}\right)$, where $m$ is a positive number. Then $\sigma_{m}^{(1)}$ is a positive measure in $R^{n}$ with finite total mass. By the definition of $\kappa$,

$$
\begin{aligned}
& \hat{\kappa}(x)=(\lambda(x))^{-i} \\
= & \left(C+\sum_{i, j=1}^{n} a_{i j} x_{i} x_{j}+\int_{|y|>0}\left(1-e^{2 \cdot \pi i x \cdot y}\right) d \sigma_{1}(y)\right)^{-1} \text { p.p., }
\end{aligned}
$$

where the symbol $\wedge$ denotes the Fourier transform. Hence

$$
\hat{\kappa}(x)\left(C+\sum_{i, j=1}^{n} a_{i j} x_{i} x_{j}+\lim _{m \rightarrow \infty} \int\left(1-e^{2 \pi i x \cdot y}\right) d \sigma_{m}^{(1)}(y)\right)=1 \text { p.p. . }
$$

that is

$$
\hat{\kappa}(x)\left(C+\sum_{i, j=1}^{n} a_{i j}^{\prime} \frac{\widehat{\partial}^{2}}{\partial x_{i} \partial x_{j}}(x)+\left(\hat{\sigma}_{m}^{(1)}(0)-\hat{\sigma}_{m}^{(1)}(x)\right)\right) \rightarrow 1 \text { p.p. }
$$

as $m \rightarrow \infty$, where

$$
a_{i j}^{\prime}=\frac{1}{4 \pi^{2}} a_{i j}
$$


Put

$$
\hat{\delta}_{m}(x)=\hat{\kappa}(x)\left(C+\sum_{i, j=1}^{n} a_{i j}^{\prime} \frac{\widehat{\partial^{2}}}{\partial x_{i} \partial x_{j}}(x)+\left(\hat{\sigma}_{m}^{(1)}(0)-\hat{\sigma}_{m}^{(1)}(x)\right)\right) .
$$

Then $\delta_{m}(x) \rightarrow 1$ p.p. as $m \rightarrow \infty$. We shall show that $\delta_{m} \rightarrow 1$ in $\mathfrak{S}^{\prime 25)}$. For any $f$ in $€,{ }^{26)}$

$$
\begin{aligned}
& \left|\hat{\delta}_{m}(x) f(x)\right| \leq C|\hat{\kappa}(x)||f(x)|+|\hat{\kappa}(x)|\left|\sum_{i, j=1}^{n} a_{i j} x_{i} x_{j}\right||f(x)| \\
+ & |f(x)|\left|\hat{\delta}_{m}^{(1)}(0)-\hat{\sigma}_{m}^{(1)}(x)\right| \\
= & C|\hat{\kappa}(x)||f(x)|+|\hat{\kappa}(x)|\left|\sum_{i, j=1}^{n} a_{i j} x_{i} x_{j}\right||f(x)|+2|f(x)| \int \sin ^{2} x \cdot y d \sigma_{m}^{(1)}(y) \\
\leq & C|\hat{\kappa}(x)||f(x)|+|\hat{\kappa}(x)|\left|\sum_{i, j=1}^{n} a_{i j} x_{i} x_{j}\right||f(x)| \\
+ & C_{1}|\hat{\kappa}(x)||x|^{2}|f(x)|+C_{2}|\hat{\kappa}(x)||f(x)|,
\end{aligned}
$$

where

$$
C_{1}=2 \int_{0<|y|<1}|y|^{2} d \sigma_{1}(y), \text { and } C_{2}=2 \int_{y \geq_{1}} d \sigma_{1} .
$$

The function $|\hat{\kappa}(x)|$ being slowly increasing, the last term of the above inequality is integrable. By Lebesgue's bounded convergence theorem,

$$
\lim _{m \rightarrow \infty} \int \hat{\delta}_{m}(x) f(x) d x=\int f(x) d x
$$

for any $f$ in $\subseteq$. Evidently the set $\left\{\delta_{m}\right\}$ is bounded in $\bigodot^{\prime}$. Hence

$$
\hat{\delta}_{m} \rightarrow 1
$$

in $\bigodot^{\prime}$ as $m \rightarrow \infty$. Therefore the distribution

$$
\delta_{m}=\left(C+\hat{\sigma}_{m}^{(1)}(0)\right)_{\kappa}+\sum_{i, j=1}^{n} a_{i j}^{\prime} \frac{\partial^{2}}{\partial x_{i} \partial x_{j}} *_{\kappa}-\kappa * \sigma_{m}^{(1)}
$$

converges to $\varepsilon$ in $\bigodot^{\prime}$.

Now suppose that $\sigma_{1} \neq 0$ in $\mathscr{C} K$. Then there exists an $m_{0}$ such that $\sigma_{m}^{(1)} \neq 0$ in $\mathscr{C} K$ for any $m \geq m_{0}$ and a function $\varphi$ of class $C^{\infty}$ with compact support such that $\varphi(x) \geq 0, S_{p} \subset \mathscr{C} K$ and

$$
\int \varphi(x) d \sigma_{m}^{(1)}(x)>0
$$

for any $m \geq m_{0}$. By the symmetricity of $\sigma_{1}$, there exists a positive number

25) $S^{\prime}$ is the space of slowly increasing distributions.

26) $S$ is the space of rapidly decreasing functions of claș $C^{\infty}$. 
such that

$$
\varphi * \sigma_{m_{0}}^{(1)}(x)>0
$$

on $\bar{B}(0 ; r)$ and

$$
(\bar{B}(0 ; r)+K) \cap S_{p}=\varnothing .
$$

By our assumption,

$$
S_{\varepsilon_{r}^{\prime}} \subset(\bar{B}(0 ; r)+K) \cap \overline{\mathscr{C} B(0 ; r)} .
$$

On the other hand the following convolutions exist and

$$
\begin{aligned}
& \delta_{m} * \varepsilon_{r}^{\prime}=\left(C+\hat{\delta}_{m}^{(1)}(0)\right) \kappa * \varepsilon_{r}^{\prime}+\sum_{i, j=1}^{n} a_{i j}^{\prime} \frac{\partial^{2}}{\partial x_{i} \partial x_{j}} * \kappa * \varepsilon_{r}^{\prime} \\
& -\kappa * \varepsilon_{r}^{\prime} * \sigma_{m}^{(1)},
\end{aligned}
$$

because $\int d \varepsilon_{r}^{\prime} \leq 1$. By the property (B'.2) of the balayaged measure,

$$
\begin{aligned}
& \delta_{m} * \varepsilon_{r}^{\prime}(\varphi)=\left(C+\hat{\delta}_{m}^{(1)}(0)\right) \int \varphi d\left(\kappa * \varepsilon_{r}^{\prime}\right) \\
+ & \sum_{i, j=1}^{n} a_{i j}^{\prime} \int \frac{\partial^{2} \varphi}{\partial x_{i} \partial x_{j}}(x) d\left(\kappa * \check{\varepsilon}_{r}^{\prime}\right)+\int \varphi * \sigma_{m}^{(1)}(x) d\left(\kappa * \check{\varepsilon}_{r}^{\prime}\right) \\
= & \left(C+\hat{\sigma}_{m}^{(1)}(0)\right) \int \varphi d \kappa+\sum_{i, j=1}^{n} a_{i j}^{\prime} \int \frac{\partial^{2} \varphi}{\partial x_{i} \partial x_{j}}(x) d \kappa \\
- & \int \varphi * \sigma_{m}^{(1)}(x) d\left(\kappa * \check{\varepsilon}_{r}^{\prime}\right) .
\end{aligned}
$$

Hence

$$
\left.\delta_{m} * \varepsilon_{r}^{\prime}(\varphi)-\delta_{m}(\varphi)\right)=\int \varphi * \sigma_{m}^{(1)}(x) d\left(\kappa-\kappa * \check{\varepsilon}_{r}^{\prime}\right)
$$

By the property $\left(B^{\prime} .1\right)$ and $\left(B^{\prime} .2\right)$ of the balayaged measure and Lemma 9 ,

$$
\lim _{m \rightarrow \infty} \int \varphi * \sigma_{m}^{(1)}(x) d\left(\kappa-\kappa * \check{\varepsilon}_{r}^{\prime}\right) \geq \int \varphi * \sigma_{m_{0}}^{(1)}(x) d\left(\kappa-\kappa * \check{\varepsilon}_{r}^{\prime}\right)>0 .
$$

on the other hand since $\delta_{m} \rightarrow \varepsilon$ in $\varsigma^{\prime}$,

$$
\delta_{m} * \varepsilon_{r}^{\prime}(\varphi)-\delta_{m}^{\prime}(\varphi) \rightarrow 0
$$

as $m \rightarrow \infty$. This is a contradiction.

Next we prove the implication (IV. 2) $\Rightarrow$ (IV. 3). Without loss of generality we may assume that $x=0$. Let $\omega$ be the closed neighborhood of 0 and $\varepsilon^{\prime}$ be the balayaged measure of $\varepsilon$ to $\mathscr{C} \omega$. By Lemma 10, we may assume that there exists a sequence $\left(\varphi_{n}\right)$ in $C_{K}$ such that it converges vaguely to $\varepsilon, \varphi_{n}(x) \geq 0$,

$$
\dot{S}_{p_{n}} \subset \omega
$$


and the sequence $\left(\varphi_{n}^{\prime}\right)$ of positive measures converges vaguely to $\varepsilon^{\prime}$, where $\varphi_{n}^{\prime}$ is the balayaged measure of the measure $\varphi_{n}$. By Theorem 2, $u_{p_{n}^{\prime}}$ being contained in $D$,

$$
S_{\varphi_{n}^{\prime}} \subset(\omega+K) \cap \overline{\mathscr{C} \omega}
$$

for each $n$. Therefore

$$
S_{\varepsilon^{\prime}} \subset(\omega+K) \cap \overline{\mathscr{C} \omega} .
$$

The implication (IV. 3) $\Rightarrow$ (IV. 1) is evident, and the proof is complete.

Similarly as Theorem $2^{\prime}$, taking for $K$ the set $\{0\}$, we immediately obtain the following

Theorem 4'. Let $D$ be a special Dirichlet space on $R^{n}$ and let $\lambda(x)$ be a negative definite function arrociated with $D$. The following conditions are equivalent.

(IV'. 1) Let $\varepsilon_{r}^{\prime}$ be the same as in Theorem 4, (IV. 1). Then

$$
\begin{gathered}
S_{\varepsilon_{r}^{\prime}} \subset \partial B(0 ; r) . \\
\lambda(x)=C+\sum_{i, j=1}^{n} a_{i j} x_{i} x_{j} .
\end{gathered}
$$

(IV'. 3) For each $x$ in $R^{n}$ and each closed neighborhood $\omega$ of $x$, let $\varepsilon_{x}^{\prime}$ be the same as in Theorem 4, (IV. 3). Then

$$
S_{\varepsilon_{x}^{\prime}} \subset \partial \omega .
$$

Next we shall consider relations between the supports of condensor measures and the support of $\sigma_{1}$. First we shall show the following two lemmas which we shall use later. Let $C_{K}^{\infty}$ be the space of functions of class $C^{\infty}$ with compact support.

Lemma 11. Let $D$ be a special Dirichlet space on $R^{n}$. Then $C_{K}^{\infty} \cap D$ is dense both in $C_{K}$ and $D$.

This is evident from the result of Deny. [4], See p. 7.

Lemma 12. Let $D$ be a special Dirichlet space on $R^{n}$ and let $\lambda(x)$ be a negative definite function associated with $D$ such that $C=\lambda(0) \neq 0$. Then for each increasing sequence $\left(\omega_{m}\right)$ of bounded open sets with $\omega_{m} \rightarrow X$, the equilibrium measure $\mu_{m}$ of $\omega_{m}$ converges vaguely to $C$ as $m \rightarrow \infty$. 
Proof. By Lemma 8 , for any $\varphi$ in $C_{K}$, there exists a potential $u_{\mu}$ in $P_{0}$ such that

$$
|\varphi(x)| \leq u_{\mu}(x)
$$

Therefore

$$
\begin{aligned}
& \int|\varphi(x)| d \mu_{m} \leq \int u_{\mu}(x) d \mu_{m}=\left(u_{\mu_{m}}, u_{\mu}\right) \\
= & \int u_{\mu_{m}}^{*}(x) d \mu \leq \int d|\mu| .
\end{aligned}
$$

That is, the set $\mu_{m}$ is vaguely bounded. Let $\mu_{0}$ be a cluster point of $\left\{\mu_{m}\right\}$. Then for each $u_{\mu}$ in $P_{0}$,

$$
\begin{aligned}
& \int u_{\mu}(x) d \mu_{0}=\lim _{m \rightarrow \infty} \int u_{\mu}(x) d \mu_{m}=\lim _{m \rightarrow \infty} \int u_{\mu_{m}}^{*}(x) d \mu \\
= & \int d \mu=\hat{\mu}(0) .
\end{aligned}
$$

On the other hand since $(\lambda(x))^{-1}$ is finite continuous, the total mass of $\kappa$ is finite. It holds that

$$
C \int u_{\Lambda}(x) d x=C \int \kappa * \mu(x) d x=C \hat{\kappa}(0) \hat{\mu}(0)=\hat{\mu}(0) .
$$

Consequently

$$
\int u_{\mu}(x) d \mu_{0}=C \int u_{\mu}(x) d x
$$

Since $P_{0}$ is total in $C_{K}$, this shows that $\mu_{0}=C$. This completes the proof.

Theorem 5. Let $D$ be a special Dirichlet space on $R^{n}$ and let $\lambda(x)$ be the negative definite function associated with $D$. Given a symmetric compact set $K$ containing 0 , the following three conditions are equivalent.

(V. 1) For each couple of two open balls $B(0 ; r)$ and $B(0 ; R)(r<R \leq+\infty)$, let $\mu=\mu^{+}-\mu^{-}$be the condensor measure associated with $B(0 ; r)$ and $\mathscr{C} \bar{B}(0 ; R)$. Then

$$
\begin{aligned}
& S_{\mu^{+}} \subset(\mathscr{C} B(0 ; r)+K) \cap \bar{B}(0 ; r), \\
& S_{\mu-} \subset(\bar{B}(0 ; R)+K) \cap \mathscr{C} B(0 ; R) .
\end{aligned}
$$

(V.2) $C=0$ and $\sigma_{1}=0$ as a measure in $\mathscr{C} K$.

(V. 3) For each couple of open sets $\omega_{1}$ and $\omega_{0}$ with disjoint closures, $\omega_{1}$ being bounded, let $\mu=\mu^{+}-\mu^{-}$be the condenisor measure associated with $\omega_{1}$ and $\omega_{0}$. Then 


$$
\begin{aligned}
& S_{\mu+} \subset\left(\mathscr{C} \omega_{1}+K\right) \cap \bar{\omega}_{1}, \\
& S_{\mu-} \subset\left(\mathscr{C} \omega_{0}+K\right) \cap \bar{\omega}_{0} .
\end{aligned}
$$

Proof. First we prove the implication (V. 1) $\Longrightarrow($ V. 2). Since $K$ is compact, $C=\lambda(0)=0$ by Lemma 12. For any $f, g$ in $C_{K} \cap D$ with $S_{f} \cap S_{g}=\varnothing$,

$$
\begin{aligned}
& (f, g)=-\frac{1}{2} \iint_{|y|>0}(f(x+y)-f(x))(\bar{g}(x+y)-\bar{g}(x)) d \sigma_{1}(y) d x \\
= & -\frac{1}{2} \iint_{|y|>0}\left(f(x+y) \bar{g}(x)+f(x) \bar{g}(x+y) d x d \sigma_{1}(y)\right. \\
= & -\frac{1}{2} \int_{|y|>0}(f * \bar{g}(x-y)+f * \bar{g}(y)) d \sigma_{1}(y) \\
= & -\int_{|y|>0} f * \bar{g}(y) d \sigma_{1}(y) .
\end{aligned}
$$

Suppose that $\sigma_{1} \neq 0$ in $\mathscr{C} K$. Then there exists a positive number $R_{1}$ such tha $\sigma_{1} \neq 0$ in $\mathscr{C}\left(\bar{B}\left(0 ; R_{1}\right)+K\right)$. By Lemma 12 , there exists a function $\varphi$ in $C_{K} \cap D$ such that $\varphi(x) \geq 0$ in $R^{n}$,

$$
S_{p} \subset \mathscr{C}\left(\bar{B}\left(0 ; R_{1}\right)+K\right) \text { and } \int \varphi(x) d \sigma_{1}(x)>0 .
$$

We take a positive number $r$ and $R$ such that

$$
0<R_{1}-R<r<R<R_{1} \text {. }
$$

Let $u_{\mu}$ be the condensor potential associated with $B(0 ; r)$ and $\mathscr{C} \bar{B}(0 ; R)$, and let $\psi$ be a function in $C_{K}$ such that $\psi(x) \geq 0$,

$$
\int \psi(x) d x=1 \text { and } S_{\psi} \subset B\left(0 ; R_{1}-R\right) .
$$

The function $u_{\mu} * \psi$ is in $C_{K}^{\infty} \cap D$ and

$$
S_{u_{\mu} * \varphi} \subset \bar{B}\left(0 ; R_{1}\right)+K
$$

Hence

$$
\left(u_{\mu} * \psi, \varphi\right)=\int \varphi(x) \mu * \psi(x) d x=0 .
$$

On the other hand, since

$$
u_{\mu} * \psi(x)=u_{\mu * \psi}(x)=1 \text { in } B\left(0 ; r-R_{1}+R\right),
$$

we obtain

$$
\int_{|x|>0} \varphi * u_{\mu} * \psi(x) d \sigma_{1}(x)>0
$$


This is a contradiction.

By Theorem 3, the implication (V.2) $\Rightarrow(\mathrm{V} .3)$ is evident.

The implication $(\mathrm{V} .3) \Rightarrow(\mathrm{V} .1)$ is evident and the proof is complete.

Similarly as Theorem $3^{\prime}$, we obtain the following

Theorem $5^{\prime}$. Let $D$ and $\lambda(x)$ be the same as in Theorem 5 . Then the following three conditions are equivalent.

$\left(V^{\prime} .1\right)$ For each couple of open balls $B(0 ; r)$ and $B(0 ; R)(r<R \leq+\infty)$, let $\mu=\mu^{+}-\mu^{-}$be the condensor measure associated with $B(0 ; r)$ and $\mathscr{C} B(0 ; R)$. Then

$$
S_{\mu^{+}} \subset \partial B(0 ; r) \text { and } S_{\mu-} \subset \partial B(0 ; R) \text {. }
$$

$$
\lambda(x)=\sum_{i, j=1}^{n} a_{i j} x_{i} x_{j}
$$

(V'.3) For each couple of open sets $\omega_{1}$ and $\omega_{0}$ with disjoint closures, $\omega_{1}$ being bounded, let $\mu=\mu^{+}-\mu^{-}$be the condensor measure associated with $\omega_{1}$ and $\omega_{0}$. Then

$$
S_{\mu+} \subset \partial \omega_{1} \text { and } S_{\mu}-\subset \partial \omega_{0}
$$

We have immediately the following application.

Proposition. Let $\lambda(x)$ be a real valued negative definite function on $R^{n}$ such that it is non-constant and $(\lambda(x))^{-1}$ is locally summable. Let $D_{a}$ be the special Dirichlet space associated with a negative definite function $\lambda_{\alpha}(x)=(\lambda(x))^{\alpha}(0<\alpha<1)^{2 \pi}$. Then there exists a symmetric positive measure $\sigma_{\alpha}$ in $R^{n}-\{0\}$ and a non-negative constant $C_{\alpha}$ such that

$$
\lambda_{\alpha}(x)=C_{\alpha}+\int_{|y|>0}\left(1-e^{2 \pi i x \cdot y}\right) d \sigma_{\alpha}(y) .
$$

Furthermore, in $D_{a}$ the followiug conditions are satisfied: (i) Let $\varepsilon_{x}^{\prime}$ be the balayaged measure $\varepsilon_{x}$ to the outside of a closed neighborhood $\omega$ of $x$. Then

$$
S_{x} \neq \partial \omega \text {. }
$$

(ii) For each couple of open sets $\omega_{1}$ and $\omega_{0}$ with disjoint closures, $\omega_{1}$ being bounded, let $\mu=\mu^{+}-\mu^{-}$be the coudensor me ure associated with $\omega_{1}$ and $\omega_{0}$. Then

$$
S_{\mu \div} \neq \partial \omega \text { and } S_{\mu-} \neq \partial \omega_{0} \text {. }
$$

27) When $\lambda(x)$ is negative definite, $(\lambda(x))^{\alpha}$ is negative definite for each $0<\alpha<1$. Cf. [4], p. 9 . 
Proof. Since $\lambda_{\alpha}(x)$ is negative definite, by Levy-Khintchine's theorem,

$$
(\lambda(x))^{\alpha}=C_{\alpha}+\sum_{i, j=1}^{n} a_{i j}^{(\alpha)} x_{i} x_{j}+\int_{|y|>0}\left(1-e^{2 \pi i x \cdot y}\right) d \sigma_{\alpha}(y) .
$$

Since $0<\alpha<1$,

$$
\frac{(\lambda(x))^{\alpha}}{|x|^{2}} \rightarrow 0
$$

as $|x| \rightarrow \infty$. Hence $a_{i j}^{(\alpha)}=0$ for any $i, j$. The conditions (i) and (ii) are evidently satisfied because $\sigma_{\alpha} \neq 0$. This completes the proof.

\section{REFERENCES}

[1] A. Beurling and J. Deny: Espaces de Dirichlet I, Le cas élémentaire, Acta Math., 99 (1958), 103-124.

[2] A. Beurling and J. Deny: Dirichlet spaces, proc. Nat. Acad. Sc. U.S.A., 45 (1959), 208-215.

[3] S. Bochner: Harmonic analysis and the theory of probablity, Berkeley, 1955.

[4] J. Deny: Sur les espaces de Dirichlet, Sem. théorie du potentiel, Paris, 1957.

[5] J. Deny: Principe complet du maximum et contractions, Colloque International du C.N.R.S., 1964.

[6] L. Schwartz: Théorie des distributions II, Paris, 1951.

\section{Mathematical Institute}

Nagoya University 